\title{
Controlled Threat Accelerates Motion Perceived Speed
}

\author{
Ahmad Yousef ${ }^{1}$ \\ 'School of Computational Science and Engineering, McMaster University, Hamilton, Ontario, Canada \\ *Correspondence: mohamas2@mcmaster.ca
}

\begin{abstract}
To presumably trigger controlled threats; we installed virtual flames in front of our moving stimuli; in conclusion, the average perceived speed is highly boosted. Lengthy exhalations can reduce the arousal and therefore putting the threat under control, see reference 3. Deep exhalations, however, boost the motion perceived speed, see reference 4 . Since our ultra-small sensor senses lengthier exhalation periods during the presentation of the virtual flames; together, we theorized that controlled but not uncontrolled threats catalyze the motion perceived speed.

Introduction

Successful activation of the retinal peripheries may change the visual awareness of the perceived speed, in another word, it speeds up the apparent motion, see reference 1,2 , and 15 . Consciously speaking, fire flames are threatening but 'under control' stimulations: thus, the acceleration of the perceived speed seems to be psychological but also neurophysiological protective action to allow humans to act timely in such critical but controlled situations. In this study, however, we tries to understand the other contributors which may boost the visual perceived speed, namely, deep exhalation, see reference 4 . Through an ultra-small pressure MEMS sensor (2 millimeters $\times 2$ millimeters) attached to a little ring and is being held near by the right nasal opening of two tested human subjects, we noticed that these subjects have lengthier respiratory exhalations when the flames are viewed; and this observation is perfectly matched with Capo, etal. 1984' findings; namely, lengthy exhalation reduces the arousal in threatening situations, see reference 3 ; in case of the dangerous situation is under control (reduced arousal). In another word, human subjects may tend to have lengthy exhalation when they try to control a dangerous situation. Astonishingly, these results return us back to the fact that the deep exhalation not only accelerates motion perceived speed but produces motion reversals, see reference 4 and 15 .
\end{abstract}

Materials \& Methods

Twelve human subjects, with dilated pupils, were recruited to participate in this study. Before each trial, it must be confirmed that each subject have a radius of pupil that's greater than 0.4 of the entire radius of the iris with the pinpoint pupil condition. Each stimulus subtends 12.4 DVA in length, and 1.72 DVA in width. Five identical fire flames, with coherent synchronized sounds, were installed in front of the moving stimulus, see reference 5 . Each human subject has to undergo into two major trials; the first trial where the stimulus moves from right to left, and the second trial moves vice versa. In each trial, human subjects have to compare the average motion perceived speed between two condition, no flames, and lots of flames. The aforementioned trials have to be counterbalanced. Each trial is repeated for three times for fine-tuning! Data Collection \& Analyses Methodology Behavioral data were collected manually, and then averaged for each subject to eventually give twelve mean values. The aforementioned mean values are then used to give cumulative estimates of the following statistical values, namely, the average and the standard error of the mean of how much perceived speed is boosted because of the installed flames. The elongation of respiratory exhalation's period that studies the effect of the controlled danger is determined by the normalized average (the data are collected from two human subjects only).

Results and Conclusion

Fire flames trigger significant acceleration in the perceived speed of the moving stimulus over the unprivileged one with normalized difference average value of acceleration $\approx 0.53$, and SEM $\approx 0.12$. This observation might suggest pupil dilation in controlled threatening situations, namely, the acceleration of the perceived speed is an indication of active retinal peripheries, and the presumed pupil dilation is to ease this active neurological process. Pupil dilation can grant greater accessibility to the retinal peripheries, thus, faster visual awareness, which seems to be so important in controlled dangerous situation. Remarkedly, Sinha et al. have astonishing findings in retinal studies, namely, they had found that the cones in the retinal peripheries signal the brain 30 milliseconds faster than the central cones. With phasic pupil dilation, the retinal peripheries will be mostly 
active in that phase; in which humans will have 'faster' visual awareness. Faster perceptual awareness assist in creating faster actions as if it is a physiological defensive mechanism to allow humans to act timely in threatening situations. However, there are a wide spectrum of events that should cause pupil dilation, namely, the dilation has the ability to estimate emotional arousal, (Bradley, 2008), represents the surprising events, (Preuschoff, 2011); and reflects individual upcoming choices and biases, (Gee, 2014). It also betrays the timing of decisions, (Einhäuser, 2010). These phasic pupil dilation in the previous occasions seems to assist the right rays of the surrounding environment to temporarily access the retinal peripheries for producing faster visual awareness, thus, humans may act in a timely fashion! Important to mention, we had been testing human subjects with tonic dilated pupils throughout all of our previous studies; because we think it's the best strategy to building a concert perspective of the theory of the two integrative conscious brains, see reference 1 and 16. It's assumed that humans had been always switching between sympathetic and parasympathetic modes in a balanced way relative to different occasions; very threatening occasions, however, could cause a significantly serious freezing perception possibly to allow fulsome awareness of the extraordinary circumstance before the action, see reference 11. The freezing perception seems to occur from difficulties in returning the pupil size to its baseline, namely, a nonphasic pupil dilation. Interestingly, spatial attention with tonic (non-phasic) dilated pupils had been reported to dilate time and space, see reference 1 . We therefore think that the spatiotemporal characteristics of the sympathetic and the parasympathetic nervous system may require some rectifications, including but not limited to clear spatiotemporal categorization between non-phasic (tonic) and phasic pupil dilation. Until we reach to settlement, we might disagree with Roelofs and colleagues perspective, namely, whether 'the freezing perception' is a parasympathetic break; because we have noticed that the freezing perception could be due to highly accelerated heart rate, see reference 4. Last but not least, the hard problem of consciousness, see reference 12 , is exceptionally raised in this study; namely, why the flames are arousal stimuli, and why they triggers such phenomenal experience. Many scientists, we think however, are moving towards the optimality to solve the hard problem of consciousness, see reference 13; namely, the answer could by beyond the physiological processes (extra physical dimensions). In another word, we think that the saccadic eye movements, in addition to the dynamics of the pupil and lens might be just assistants to the main contributor in generating the visual awareness which is assumed to be perfect entanglement between each photon of the external world, and its corresponding retinal photoreceptors; an outstanding example is given in reference 17.

Essential Notifications

Full details about the nasal pressure sensor and installation procedures are available upon request. Important to mention, according the two tested human subjects, the normalized average value of the elongation of the respiratory exhalation's period in this 'controlled danger' study $\approx 0.463$, and SEM $\approx 0.083$. Readers should have direct access to the stimuli, see reference 4. Advisably, download the videos for the best quality. For rapid validation process, have the test with dilated pupil, watch the videos in moderate environment; namely, fifty percent of the monitor's max. brightness. Caffeine intake also dilate the pupil, in case of pinpoint pupils, see reference 14. Scholars with brain imaging facilitations are welcome to collaborate in challenging investigations, namely, to achieve critical analyses of the spatiotemporal properties of the hMT+ region. Enquiries may be sent to the author.

\section{Transactional References}

[1] Yousef, A. 2019. "Spatial Attention Dilates Time and Length." PsyArXiv. doi:10.31234/osf.io/qazj9.

[2] Traschütz etal., 2012. Speed change detection in foveal and peripheral vision. Vision Research

[3] Cappo, etal., 1984. The utility of prolonged respiratory exhalation for reducing physiological and psychological arousal in non-threatening and threatening situations. J Psychosomatic Research.

[4] Yousef, Ahmad. 2019. "Deep Breathing Alters Visual Motion Perception." PsyArXiv. doi:10.31234/osf.io/up3sa.

[5] You may find the stimuli in the following link:

https://drive.google.com/drive/folders/1tJmtt3lfZpHk6VQ2WzldkXeGtMC8rh0g

[6] Sinha, etal. (2017). Cellular and circuit mechanisms shaping the perceptual properties of the primate fovea. Cell Journal.

[7] Bradley, (2008). The pupil as a measure of emotional arousal and autonomic activation. Psychophysiology.

[8] Einhäuser, Koch, and Carter (2010). Pupil dilation betrays the timing of decisions. Front. Human Neuroscience.

[9] Preuschoff, etal. (2011). Pupil dilation signals surprise: evidence for noradrenaline's role in decision making. Frontiers in Neuroscience.

[10] de Gee (2014). Decision-related pupil dilation reflects upcoming choice and individual bias. PNAS.

[11] Roelofs K. (2017). Freeze for action: neurobiological mechanisms in animal and human freezing. Philosophical transactions of the Royal Society: Biological sciences.

[12] David Chalmers, (1995). "Facing Up to the Problem of Consciousness." Journal of consciousness studies.

[13] Yousef, A. 2019. "Consciousness Might Be Localized in Extra Physical Dimensions." PsyArXiv. doi:10.31234/osf.io/angc8.

[14] Abokyi, 2017.Caffeine intake associated with pupil dilation \& enhanced

accommodation. Eye Journal.

[15] Yousef, 2019. "Retinal Peripheries Generate Illusory Motion Reversals." PsyArXiv. doi:10.31234/osf.io/gfmpe.

[16] Yousef. 2019. "Two Distinct Fusiform Face Areas." PsyArXiv.

doi:10.31234/osf.io/a8gzv.

[17] Yousef. 2019. "Motion Induced Colours Fusion." PsyArXiv. doi:10.31234/osf.io/mkth2. 